\title{
An Analysis of Neonatal Morbidity and Mortality in Azare, North- Eastern Nigeria.
}

\author{
Imoudu IA,Ahmad H, Yusuf MO,Makarfi HU, Umara T. \\ Department of Paediatrics, Federal Medical Centre, Azare, Bauchi state.
}

\begin{abstract}
An Analysis Of Neonatal Morbidity And Mortality In Azare, North-Eastern Nigeria.
Background: Mortality occurring among babies less than 28 days of life comprises about $40 \%$ of all under-five deaths. Improvement on neonatal statistics is thus crucial to the attainment of the fourth millennium development goal (MDG).

Objective: To describe the pattern of morbidity and mortality of in-patients as seen in the Special Care Baby Unit of the Federal Medical Centre Azare, North-eastern Nigeria over a 12-month period.

Methods: Retrospective data of sequential admissions into the SCBU from 1st January to 31 st December 2012 were analysed.

Results: A total of 328 babies were admitted in the SCBU during the study period. Of these 179(54.6\%) were males and 149(45.6\%) females. Major causes of admission were neonatal sepsis with 108 (32.9\%), prematurity 78 (23.8\%), and Perinatal asphyxia 62 (18.9\%). Two hundred and eight (63.4\%) were discharged, and $17(5.2 \%)$ discharged against medical advice (DAMA). The mortality rate was $25.9 \%$ (85 patients), with prematurity accounting for 27 (31.8\%), and perinatal asphyxia 23 (27.1\%) of the mortalities.

Conclusion: There is a high neonatal mortality rate as well as high case fatality rates from largely preventable diseases. Improvement can be achieved by accelerated action in the context of Integrated Maternal, Newborn and Child Health (IMNCH) strategy and MDGs' objectives.
\end{abstract}

Keywords: Pattern, Morbidity, Mortality, Admissions, Outcome.

\section{Introduction}

Even as some progress is being made and neonatal mortality rates are declining in all regions of the world, this is largely distributed disproportionately. More than half (53\%) of all neonatal deaths occur in only five countries: India, Nigeria, Pakistan, China and Democratic Republic of the Congo. ${ }^{1-3}$ Mortality occurring amongst babies less than 28 days of life comprises about $40 \%$ of all under-five deaths. ${ }^{2}$ Improvement on neonatal statistics is thus crucial to the attainment of the fourth millennium development goal (MDG). The periodic evaluation of morbidity and mortality is critical to improving quality of service delivery hence we have opted to determine the trend of morbidity and mortality in the special care baby unit (SCBU) of the Federal Medical Centre, Azare as an audit. The Special Care Baby Unit provides services for the populations of Bauchi, Jigawa and Yobe states in northern- Nigeria. To the best of our knowledge, no such study has been carried out previously in this unit since its inception in 2004.

\section{Objective}

To determine the pattern of morbidity and mortality in the neonatal age group amongst in-patients of the Federal Medical Centre (FMC)Azare, Bauchi state, North-eastern Nigeria over a 12-month period.

\section{Methods}

Retrospective data of sequential admissions into the SCBU from 1st January to 31st December 2012 were analysed. Information obtained included; biodata, diagnosis, duration of hospital stay and outcome such as discharge, referrals, Discharge Against Medical Advice(DAMA) and deaths. The obtained Data was entered into the Statistical Package for Social Sciences (SPSS) version 17 for analysis.

\section{Results}

The total number of admissions during the period under review was 328. Females accounted for 149 (45.4\%) of this figure and males made up 179 (54.6\%),giving a male to female ratio of 1.2: 1 . This is displayed in figure 1. Eighty-five patients died while on admission giving a mortality rate of $25.9 \%$. Two hundred and eight patients (63.4\%) were discharged while 17 (5.2\%) were discharged against medical advice (DAMA). Figure 2 illustrates these findings.

Figure 1. Sex distribution 


\section{SEX DISTRIBUTION}
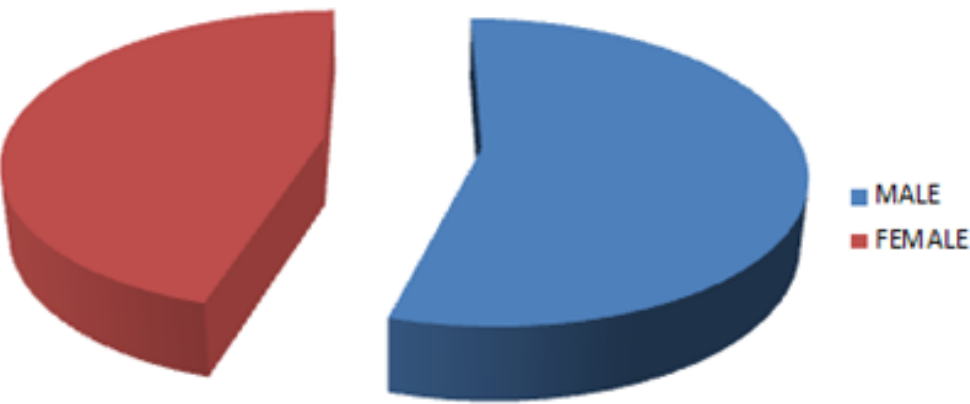

Figure 2. Distribution of outcome

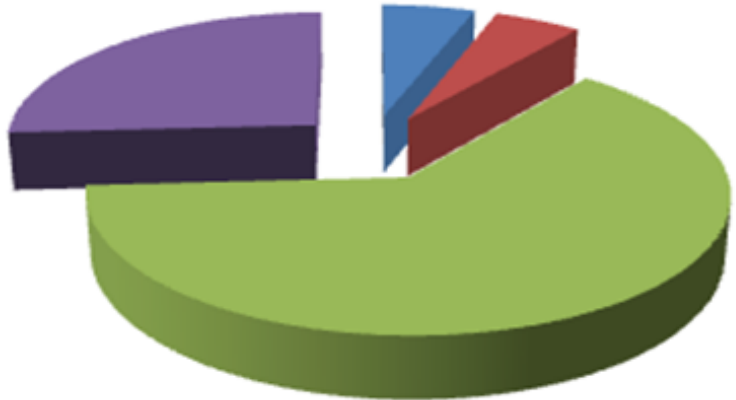

Figure3. Morbidity pattern.

The major causes of admission were perinatal asphyxia, Sixty-two patients (18.9\%), neonatal sepsis, $108(32.9 \%)$, neonatal hyperbilirubinaemia, 11 patients $(3.4 \%)$ and prematurity, $78(23.8 \%)$.Neonatal tetanus accounted for $4(1.2 \%)$ of the total admissions while others (congenital anomalies, birth trauma, macrosomia, dehydration fever, haemorrhagic disease of the newborn and merconium aspiration syndrome) constituted 65 $(19.8 \%)$ admissions into SCBU. Figure 3 demonstrates the morbidity pattern.

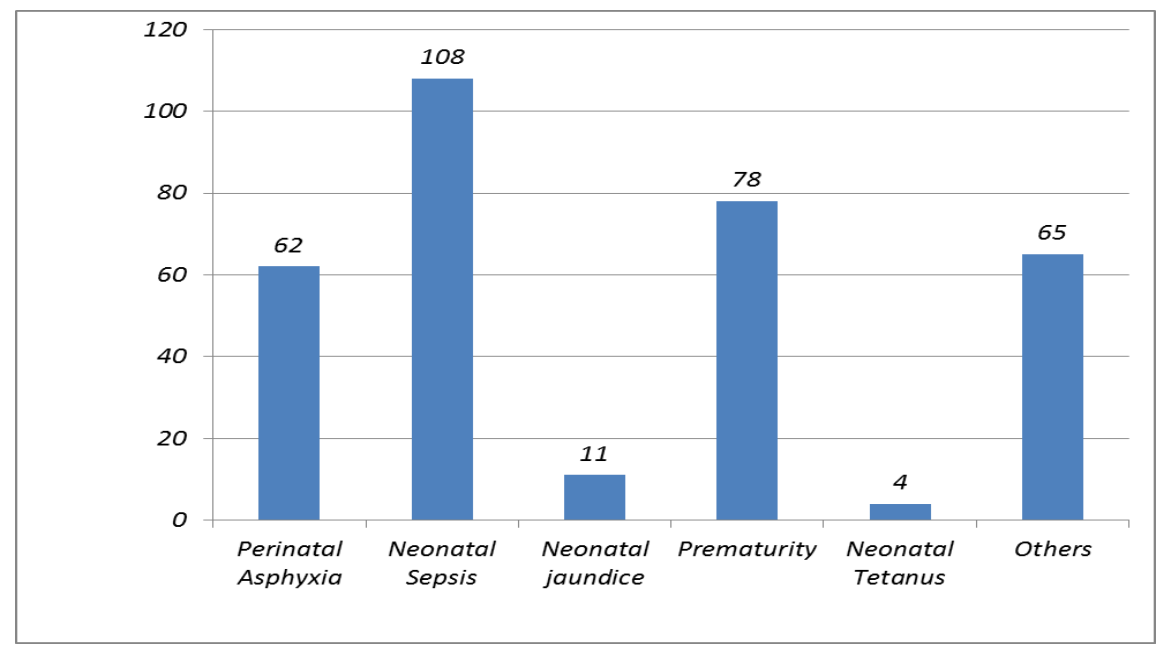

Others; congenital anomalies, birth trauma, macrosomia, dehydration fever, haemorrhagic disease of the newborn, merconium aspiration syndrome.

Prematurity accounted for the highest number of mortalities $27(31.8 \%)$ giving a case fatality rate of $34.5 \%$. Perinatal asphyxia constituted $23(27.1 \%)$ with a case fatality rate of $37.1 \%$, neonatal sepsis made up 13 $(15.3 \%)$ of the mortalities as well as a case fatality rate of $12.0 \%$. Neonatal tetanus accounted for $3(3.5 \%)$ of the mortalities along with a case fatality rate of $75 \%$. Table I shows these findings. 
An Analysis Of Neonatal Morbidity And Mortality In Azare, North- Eastern Nigeria.

Table I.Distribution of mortalities by diagnosis.

\begin{tabular}{lllll}
\hline Disease & No. of Admissions & No. of Deaths & Percentage Deaths (\%) & Case fatality rate (\%) \\
\hline Neonatal sepsis & 108 & 13 & 15.3 & 12.0 \\
Prematurity & 78 & 27 & 31.8 & 34.6 \\
Perinatal asphyxia & 62 & 23 & 27.1 & 37.1 \\
Neonatal Jaundice & 11 & 6 & 7.0 & 54.5 \\
Others & 51 & 13 & 15.3 & 25.5 \\
Neonatal tetanus & 4 & 3 & 3.5 & 75.0 \\
Total & 328 & 85 & 100 & \\
\hline
\end{tabular}

\section{Discussion}

The number of patients admitted into our SCBU within the one-year period is 328 . Three hundred and fifty-six admissions were recorded over a similar period at Olabisi Onabanjo University Teaching Hospital, Sagamu, Ogun state in 2012. ${ }^{5}$ Conversely, Ekwochi et al documented 126 admissions over a 5-month period in Enugu. ${ }^{6}$ The number of admissions recorded in our study is lower than the 356 documented in Sagamu. This could have resulted from the lower population of the catchment area of Federal Medical Centre (FMC), Azare when compared to that of the Olabisi Onabanjo University Teaching Hospital, Sagamu. Another contributing factor could be the lower level of uptake of neonatal health care services by the population within the catchment of FMC Azare.

Neonatal Mortality Rate in this study was $25.9 \%$. This is significantly higher than the $19.3 \%$ documented in Calabar in 2008, ${ }^{7}$ the $16.9 \%$ recorded in Kano, ${ }^{8}$ the $15.4 \%$ recorded in Sagamu ${ }^{3}$ as well as the $19.8 \%$ documented in Enugu. ${ }^{6} \mathrm{~A}$ study conducted in the Gambia however, reported a neonatal mortality rate of $39 \%$. The high mortality rate obtained in our study is likely a consequence of the fact that most of the deliveries in the areas covered by the hospital still take place at home where the conditions are not optimal for neonatal health care. ${ }^{10}$ In addition, patients often present late to the hospital further compromising their chances of survival. Discharge against medical advice (DAMA) rate was $5.2 \%$ in the period under review. This is higher than the $4.3 \%$ documented in a neonatal unit in Port Harcourt, ${ }^{11}$ but lower than the $6.7 \%$ reported in Gwagwalada, Nigeria. ${ }^{12}$ The DAMA rate in the present study almost certainly mirrors the level of poverty and disposition towards the use of alternative sources of medical care in the covering communities. ${ }^{10}$

The prominent diagnoses among admitted patients in the present study are neonatal sepsis, prematurity, perinatal asphyxia, neonatal hyperbilirubinaemia and neonatal tetanus. This is akin to findings from other parts of Nigeria. ${ }^{5,6-8}$ Neonatal sepsis accounted for the most admissions in our study and is symptomatic of the prevailing situation of poor antenatal care, lack of skilled birth attendants, and inadequate newborn care.

Prematurity is the most predominant cause of mortality in this study. This is similar to findings in Sagamu. ${ }^{5}$ However, some other Nigerian studies have indicated neonatal sepsis and perinatal asphyxia as the prime contributors to neonatal mortality. ${ }^{6,7,8}$ Limitations in facilities and manpower largely contributed to the high mortality of prematurity in the present study. The highest case fatality rate of $75 \%$ was nevertheless recorded for neonatal tetanus with neonatal hyperbilirubinaemia constituting the second highest case fatality rate of $54.5 \%$. Neonatal sepsis had a case fatality rate of $12.0 \%$. Neonatal tetanus also accounted for the highest case fatality rate of $33.3 \%$ in Sagamu, ${ }^{5}$ a figure which is significantly lower than the $75 \%$ in this study. The high case fatality rate of neonatal tetanus in this study is the aftermath of late presentation, harmful traditional practices and inadequate facilities. It also emphasizes the need to focus on the simple but effective preventive measures available for the management of neonatal tetanus.

\section{Conclusion}

There is a high neonatal mortality rate as well as high case fatality rates for largely preventable diseases in this study. The foremost causes are similar to findings in other Nigerian health facilities. Strategies to stem this trend would include boosting immunization coverage and uptake, improving antenatal and perinatal care coupled with enhancement of health education campaigns. Some of these newborn lives could be saved with the existing policies if the cost was more affordable. Expanding the coverage of existing policies with accelerated action in the context of the Intergrated Maternal, Newborn and Child Health strategy (IMNCH) and MDGs' objectives is an effective way to accomplishing significant reduction in neonatal morbidity and mortality. 


\section{References}

[1] Lawn JE, Cousens S, Zupan J. Four million neonatal deaths: when? where? why? Lancet 2005; 365: 891-900.

[2] Saving newborn lives in Nigeria: Newborn health in the context of the Intergrated Maternal, Newborn and Child Health Strategy. Abuja: Federal Ministry of Health, Save the Children, ACCESS. 2009;8-11.

[3] World Health Organization. Maternal, newborn, child and adolescent health programme. (cited 2013 July 4). Available from: www.who.int/maternal_child_adolescent/epidemiology/newborn/en/.

[4] United Nations Inter-agency Group for child Mortality Estimation. Levels and trends in child mortality. Annual report 2011 (document on the internet). Healthy Newborn Network; (cited 2013 July 4). Available from: http://www.unicef.org/media/media_59795html.

[5] Ayeni VA, Oladipo AO, Ogunlesi TA, et al. Morbidity and mortality pattern among in-patients in the paediatric department of Olabisi Onabanjo University Teaching Hospital over a twelve-month period. Paper presented at: The Annual General and Scientific Meeting of the Paediatric Association of Nigeria; 2013 January 22-26; Enugu, Nigeria.

[6] Ekwochi U, Ezenwosu OU, Nwokoye IC, Ndu IK. Pattern of morbidity and mortality of admissions into the special care baby unit of Enugu State University Teaching Hospital, Parklane, Enugu, South- East Nigeria. Paper presented at: The Annual General and Scientific Meeting of Paediatric Association of Nigeria; 2013 January 22- 26: Enugu, Nigeria.

[7] Udo JJ, Anah MU, Ochigbo SO, Etuk IS, Ekanem AD. Neonatal morbidity and mortality in Calabar, Nigeria: a hospital-based study. Niger J Clin Pract 2008; 11 (3): 285-89.

[8] Mukhtar-Yola M, Iliyasu Z. A review of neonatal morbidity and mortality in Aminu KanoTeaching Hospital, Northern Nigeria. Trop Doct 2007; 37(3): 130-2.

[9] Leach A, McArdle TF, BanyaWAS,et al. Neonatal mortality in a rural area of The Gambia. Ann Trop Paediatr 1999; 19: 33-43.

[10] Udoma EJ, Udo JJ, Etuk SJ, Duke ES. Morbidity and mortality among infants with normal birth weight in a newborn baby unit. Niger J Paed 2001; 28: 13-17.

[11] Opara P, Eke G. Discharge against medical advice amongst neonates admitted into a special care unit in Port Harcourt, Nigeria. The Internet Journal of Pediatrics and Neonatology 2010; 12 (2). (cited 2013 July 10). Available from: http://archive.ispud.com:80/journal/the-internet-journal-of-pediatrics-and- neonatology/volume-12-number-2/discharge-againstmedical-advice-amongst-neonates-admitted-into-a-special-care-baby-unit-in-port-harcourt-nigeria.html.

[12] Okechukwu AA. Discharge against medical advice in children at University of Abuja Teaching Hospital, Gwagwalada, Nigeria. J Med. Med. Sci 2011; 2 (7): 949-54 